\title{
LiCl-KCl eutectic molten salt as an original and efficient medium to intercalate metals into graphite: case of europium
}

\author{
Mickaël Bolmont $^{\mathrm{a}}$, Sébastien Cahen ${ }^{\mathrm{a}}$, Mélissa Fauchard ${ }^{\mathrm{a}}$, Romain Guillot ${ }^{\mathrm{a}}$, \\ Ghouti Medjahdi $^{\mathrm{a}}$, Pascal Berger ${ }^{\mathrm{b}}$, Gianrico Lamura ${ }^{\mathrm{c}}$, Philippe Lagrange ${ }^{\mathrm{a}}$, Claire Hérold $^{\mathrm{a}}$ \\ anstitut Jean Lamour, UMR UL-CNRS 7198, \\ ${ }^{\mathrm{a}}$ Faculté des Sciences et Technologies, B.P. 70239, 54506 Vandouvre-lès-Nancy Cedex, France. \\ ${ }^{b}$ NIMBE, CEA, CNRS, Université Paris-Saclay, CEA Saclay 91191 Gif sur Yvette Cedex, France. \\ ${ }^{c}$ CNR-SPIN, Corso Perrone 24, 16152 Genova, Italy.
}

\begin{abstract}
We present the successful intercalation of europium into graphite by using an original method based on $\mathrm{LiCl}-\mathrm{KCl}$ molten salts mixture. After solubilization of europium in the liquid chlorides mixture at $450{ }^{\circ} \mathrm{C}$, reactivity toward graphite is investigated. We evidence the formation of the first stage $\mathrm{EuC}_{6}$ Graphite Intercalation Compound (GIC) with a repeat distance $\mathrm{Ic}=487 \mathrm{pm}$. Using $00 l \mathrm{X}$-ray diffraction and ion beam analysis, we show that europium is intercalated into the bulk with remarkable degree of homogeneity. This is also confirmed by magnetic measurements that clearly put in evidence the antiferromagnetic transition at about $\mathrm{T}_{\mathrm{N}}=40 \mathrm{~K}$. Below such temperature the characteristic step-like behavior of metamagnetic transitions was found by isothermal magnetization measurements, that is generally visible only in highly pure samples.
\end{abstract}

\section{Introduction}

Considered as model materials in order to probe the relationship between structure and physical properties, graphite intercalation compounds (GICs) have been the subject of numerous studies and books [1,2]. During the eighties, the preparation of metal-based GICs has mainly been realized by performing intercalation reactions of alkali, alkaline-earth and lanthanide metals

\footnotetext{
*Corresponding author:Tel: +33383684645.E-mail: sebastien.cahen@univ-lorraine.fr
} 
into graphite. These GICs were studied due to their specific physical properties, especially electrical resistivity [3-5], magnetism [6-8] and superconductivity [9-12]. If some synthesis routes involving direct reaction have been investigated using electrochemical intercalation [13-15], high pressure reactions [16,17] or ball-milling synthesis [18], most of the past intercalation reactions were performed by direct action of the metal vapor. Nevertheless, such techniques do not allow preparing bulk intercalation compounds with lanthanides [19-21], even if some positive results could have been obtained using very small graphite single crystals [22].

In order to prepare bulk GICs, the possibility to synthesize novel binary or ternary donor-type GICs has been investigated, mainly using the lithium-based molten alloy method [23,24]. In this solid-liquid way of synthesis, the alkali metal, lithium in this case, plays the role of an intercalation vector for electropositive metals such as calcium, barium or europium. In the graphite-lithium-calcium system, $\mathrm{Li}_{3} \mathrm{Ca}_{2} \mathrm{C}_{6}$ and $\mathrm{Li}_{0.5} \mathrm{Ca}_{3} \mathrm{C}_{6}$ ternary compounds are evidenced together with the binary $\mathrm{CaC}_{6}$ GIC [25-27]. On the contrary in the graphite-lithium-barium system, only the $\mathrm{BaC}_{6}$ binary compound is obtained [23]. Finally in the graphite-lithiumeuropium system, one ternary compound $\left(\mathrm{Li}_{0.2} \mathrm{Eu}_{2} \mathrm{C}_{6}\right)$ and the binary $\mathrm{EuC}_{6}$ can be prepared [28-30]. It should be noted that for $\mathrm{EuC}_{6}$, a significant amount of lithium metal (>10\% at.) is observed in the samples which are clearly heterogeneous. This first limitation for preparing homogeneous samples is one of the drawbacks of the solid-liquid method. Another one is pointed out regarding the intercalation of different metals especially lanthanides which remains unsuccessful. In the specific case of the intercalation of magnetic rare earth metals into graphite, it is interesting to notice the paper of Hagiwara et al. who claimed the possible synthesis of various $\mathrm{LnC}_{6}$ GICs using a $\mathrm{Ln}-\mathrm{LnCl}_{3}-\mathrm{LiCl}-\mathrm{KCl}$ mixture [31]. Unfortunately, this work reports the systematic presence of complex chlorides $\mathrm{K}_{\mathrm{x}} \mathrm{Ln}_{\mathrm{y}} \mathrm{Cl}_{\mathrm{z}}$ in the obtained samples together with a lack of crystallographic data and an unclear reactional mechanism. However, the general idea to proceed to intercalation reaction using a molten salt medium could be promising compared to the solid-liquid method we developed in alkali-based molten alloys. Indeed, the molten medium is only considered as an inert solvent versus graphite, so that undesired intercalation should be limited. Moreover, the chosen medium is clearly different as it consists in an ionic salt instead of a metallic alloy, so that other reactional mechanisms are expected. Finally, a method allowing the intercalation of europium into the bulk of pyrolytic graphite is still not available. Successfully developing such a synthesis route would be a milestone for the possible intercalation of elements hardly intercalated alone into graphite. 
In this paper, we present the original synthesis in the $\mathrm{LiCl}-\mathrm{KCl}$ molten medium of the well-known $\mathrm{EuC}_{6}$ binary intercalation compound, which has been largely characterized by specific $00 l \mathrm{X}$-ray diffraction, nuclear microprobe analysis and dc magnetometry.

\section{Experiment}

The individual $\mathrm{LiCl}$ and $\mathrm{KCl}$ chlorides have been outgassed for 24 hours at $240{ }^{\circ} \mathrm{C}$ under secondary vacuum $\left(10^{-6} \mathrm{mbar}\right)$. Then, in a glove box under pure argon atmosphere $(<1 \mathrm{ppm}$ in $\mathrm{O}_{2}$ and $\mathrm{H}_{2} \mathrm{O}$ ) the $\mathrm{LiCl}-\mathrm{KCl}$ mixture has been precisely weighed in order to reach the eutectic composition $(59.2 \%$ mol. in $\mathrm{LiCl})$ reported on the corresponding binary phase diagram [32]. The eutectic mixture is introduced in a stainless steel reactor and heated up to $450{ }^{\circ} \mathrm{C}$ in order to achieve its melting. Then, $2 \%$ mol. of europium (Aldrich, purified by distillation $99.99 \%$ ) is dissolved in the molten medium which is mechanically homogenized. The amount of metal is chosen in order to perform the reaction with a large excess of metal compared to the quantity of graphite. This host material is a pyrolytic platelet of graphite $\left(10 \times 2 \times 0.5 \mathrm{~mm}^{3}\right)$ hanged up a tungsten sample-holder. The reactor airtight closed is placed in a furnace and the reaction is carried out at $450^{\circ} \mathrm{C}$ for 6 days outside the glove box. After reaction, the reactor is open inside the glove box and heated again for extracting the intercalated sample. The latter is then hot centrifuged in order to remove the excess of condensed chlorides from its surface and finally conditioned in various airtight sample-holders depending on the subsequent characterization method (XRD or magnetic measurements). For ion beam analysis, samples are quickly air transferred from inert atmosphere to the nuclear microprobe instrument, what is not critical for the chemical analysis of the bulk GIC.

X-ray diffraction (XRD) experiments are realized with a Bruker D8 diffractometer using molybdenum $K_{\alpha 1}$ radiation $\left(\lambda_{K \alpha 1}=70.926 \mathrm{pm}\right)$ with a Lynxeye detector. The sample is sealed in a glass capillary placed so that the incident X-ray beam is parallel to the $a b$ graphite planes. It is so possible to record the $00 l$ reflections separately. After acquisition, a quantitative analysis of these Bragg peaks is performed for the determination of the stacking sequence of the intercalated layers by calculation of the one-dimensional electronic density profile along the c-axis [33].

DC magnetometry was performed using a commercial superconducting quantum interference device (SQUID) MPMS2 by Quantum Design as detailed in the Supplementary Material (SM). 
Nuclear microprobe analyses are performed using a highly energetic proton ion beam (about $3000 \mathrm{keV}$ ) interacting with a target constituted of the analyzed GIC. The interaction of the ion beam with the electrons of the target sample leads to a progressive slowdown of proton until they stop. This electronic stopping power of the target defines the probed thickness.

The interaction between target nuclei and the ion beam is used to measure local elementary compositions in depth and laterally. Analyzed windows are large $\left(250 \times 250 \mu \mathrm{m}^{2}\right)$ with a micrometric resolution due to the spot size of $3.5 \times 4 \mu \mathrm{m}^{2}$. The ion beam analysis is of course performed in order to confirm the expected $\mathrm{EuC}_{6}$ composition, but also for probing the homogeneity of the sample and the absence of residual lithium. In order to realize the elementary titration, two kinds of interactions are taken in consideration, i.e. elastic scattering and nuclear reactions which are interpreted by Rutherford Back-Scattering (RBS) and Nuclear Reaction Analyses (NRA) techniques respectively. Considering the ion beam energy, europium is determined by elastic scattering leading to absolute concentration without the need of a reference sample. For lithium and carbon, the use of a proton ion beam instead of more usual ${ }^{4} \mathrm{He}$ one enables to favor an enhanced backscattering signal on carbon and lithium (with socalled non-Rutherford cross-sections). Finally, in the conditions of our experiment $(3000 \mathrm{keV}$, back detector at a $170^{\circ}$ angle from the direction of the incident beam), a specific nuclear reaction between proton beam and lithium-7 occurs at a very high energy discriminated from the reaction with the heavier $\mathrm{C}$ and $\mathrm{Eu}$ elements. It is finally possible to fit as best as possible the experimental spectrum with a simulated one using the SimNRA software [34]. The calculated spectrum is issued from the superimposed individual contributions of all the interactions of the proton beam with each isotope of each element present in the different sublayers of the defined solid.

\section{Results and discussion}

Optimal experimental conditions can be investigated by modifying the amount of europium in the mixture, the temperature and duration of the intercalation reaction. If the influence of the amount of Eu and reaction temperature still need to be further investigated, we have determined that the thermodynamic equilibrium was reached after 6 days of reaction at $450{ }^{\circ} \mathrm{C}$ in the $\mathrm{LiCl}-\mathrm{KCl}$ medium in which $2 \%$ mol. of metallic europium are dissolved. The graphite platelet is extracted from the reactive mixture then separated from its tungsten sample-holder. After 
evacuation of the excess of chlorides condensed on the surface, the final sample is a shiny silver rectangular platelet, a color clearly attributed to a bulk $\mathrm{EuC}_{6}$ compound [29].

\subsection{Structural analysis of the EuC 6 bulk intercalated compound}

Thanks to the oriented texture of pristine PGCCL, it is possible to record $00 l \mathrm{X}$-ray diffraction pattern specifically. The corresponding diffractogram is presented Fig.1.

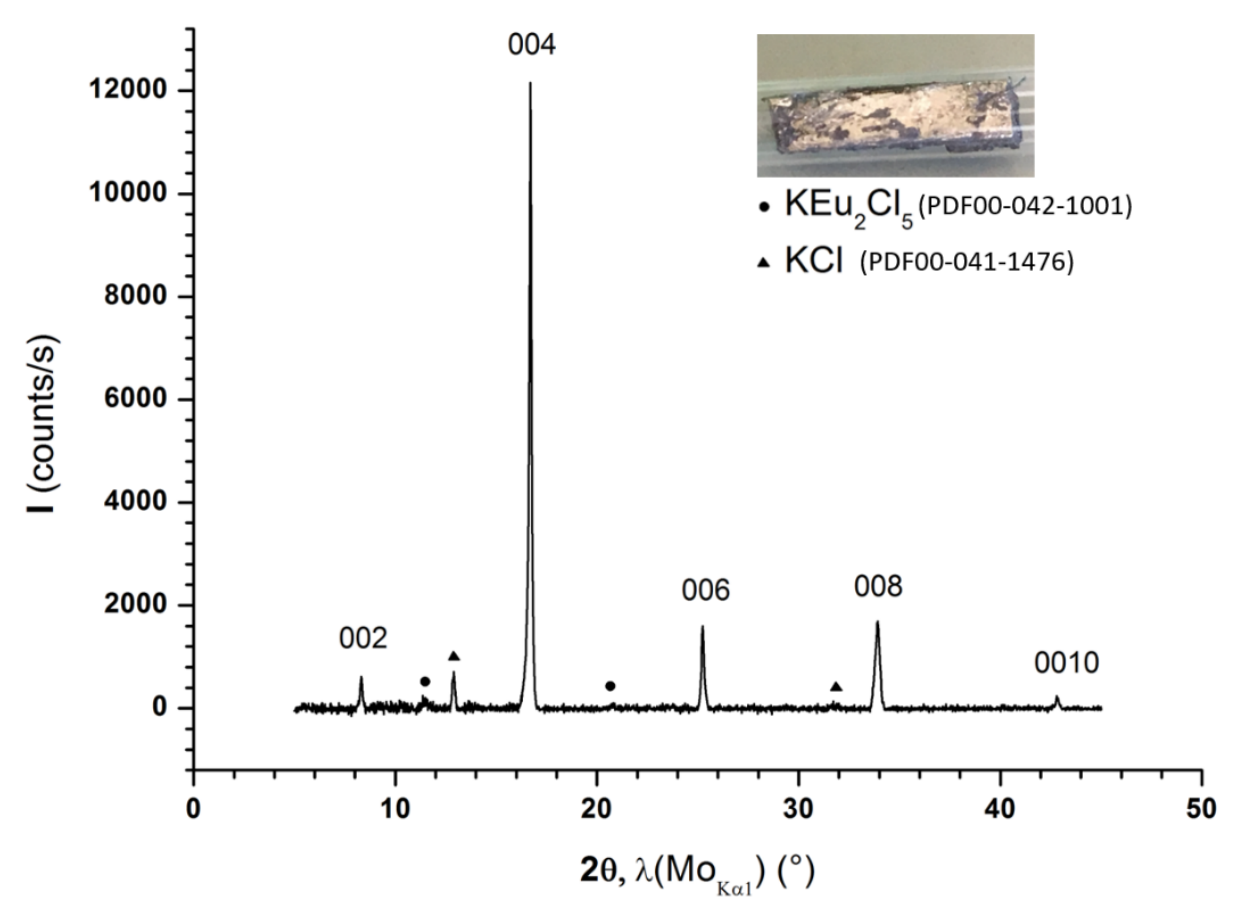

Figure 1 - 00l X-ray diffraction pattern of the first stage EuC6GIC

$\left(\mathrm{Ic}=487 \mathrm{pm}, \lambda_{\mathrm{MoKa} 1}=\mathbf{7 0 . 9 2 6 \mathrm { pm }}\right)$. A picture of the bulk sample is given in inset.

This diagram shows intense and individualized $00 l$ reflections which correspond with a $1^{\text {st }}$ stage $\mathrm{EuC}_{6}$ GIC with a repeat distance $\mathrm{Ic}=487 \mathrm{pm}$ perfectly matching the known crystallographic data [22]. No reflection due to graphite or other intercalation compound can be observed. Only small reflections due to superficial $\mathrm{KCl}$ salt are observed, but with very low intensities. Two other small diffraction lines are also visible at $2 \theta=11.4^{\circ}$ and $20.7^{\circ}$ respectively, and are explained by the residual presence of a $\mathrm{KEu}_{2} \mathrm{Cl}_{5}$ complex [35] on the surface of the sample. This result implies the possible formation of divalent $\mathrm{Eu}^{+\mathrm{II}}$ ions in the molten medium, what is in agreement with the formation of the $\mathrm{EuC}_{6}$ compound. Indeed, ${ }^{151} \mathrm{Eu}$ Mössbauer studies 
performed on $\mathrm{EuC}_{6}$ showed the presence of divalent ions in the van der Waals galleries [36,37]. Compared to the XRD pattern of the bulk intercalation compound obtained using the solid-liquid method [29], it is very interesting to notice that no europium inclusion (110 diffraction peak at $2 \theta=12.48^{\circ}$ ) is detected using this molten salts method. Considering the absence of other diffraction peaks due to any inclusion, and considering the sensibility of XRD analysis, the final sample corresponds to almost $95 \%$ vol. of $\mathrm{EuC}_{6}$ phase.

By quantitative analysis of the $00 l$ diffraction pattern, the electronic density profile has been calculated in order to confirm the synthesis of $\mathrm{EuC}_{6}$. The profile determined from the aforementioned experimental data is compared with the one calculated considering one plane of europium atoms intercalated into graphite. This comparison is presented Fig. 2 and Table 1 reports the experimental and calculated data.

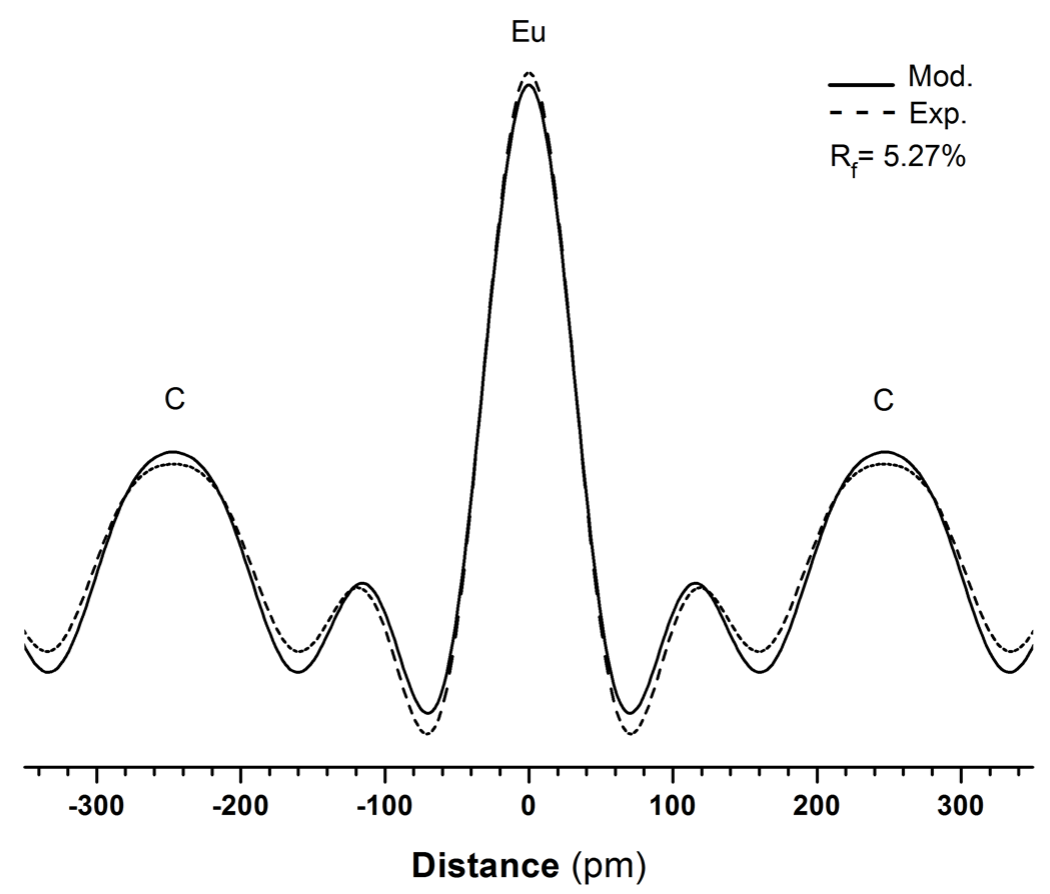

Figure 2 - 1D $c$-axis electronic density profiles of the $\mathrm{EuC}_{6}$ compound. Model and experiment are drawn in solid and dotted lines, respectively. 
Table 1 - Experimental and calculated structure factors of the $00 l$ reflections used for the calculation of the electronic density profiles of $\mathrm{EuC}_{6}$.

\begin{tabular}{|c|c|c|c|c|c|}
\hline $00 l$ & $\theta\left({ }^{\circ}, \lambda M_{K \alpha l}\right)$ & $\mathrm{d}_{00 l}(p m)$ & $\mathrm{I}_{\mathrm{C}}(p m)$ & $\mathrm{F}_{00 l}$ exp. & $\mathrm{F}_{00 l}$ calc. \\
\hline 002 & 4.15 & 489.5 & 490 & 34.4 & 38.3 \\
004 & 8.35 & 244.3 & 489 & 100.0 & 100.0 \\
006 & 12.62 & 162.3 & 487 & 52.9 & 41.6 \\
008 & 16.96 & 121.6 & 486 & 70.1 & 70.0 \\
0010 & 21.39 & 97.2 & 486 & 34.3 & 34.3 \\
\hline
\end{tabular}

Using five Bragg reflections for the calculation, the model is in good agreement with one single layer of europium between two graphene sheets, with a C/Eu atomic ratio equal to 6 and a distance between layers equivalent to the half of the repeat distance. In the model, the latter is equal to $487 \mathrm{pm}$ in agreement with the value of $487.6 \mathrm{pm}$ calculated from the experimental data. The residual factor calculated by least-square method using these data is $\mathrm{R}_{\mathrm{F}}=5.3 \%$, confirming the nice agreement between experimental and calculated data. This result clearly confirms that we succeeded in preparing a bulk $\mathrm{EuC}_{6}$ compound using a novel route.

\subsection{Ion beam analysis}

In order to probe the homogeneity of the synthesized GIC and to confirm the chemical composition issued from the X-ray diffraction data, measurements have been performed by ion beam analysis. Such a technique used for GICs has already demonstrated numerous advantages as the possibility to realize the simultaneous titration of all the elements of the periodic table, its reliability and the possibility to analyze the chemical composition of a sample laterally as well as in its depth [38-40]. A little residue of $\mathrm{KCl}$, evidenced by XRD (Fig.1), remains on the surface on the bulk sample after synthesis. So, the ion beam analysis has been performed on a freshly cleaved sample in order to probe the bulk sample. The corresponding spectrum and its simulation are drawn Fig.3. 


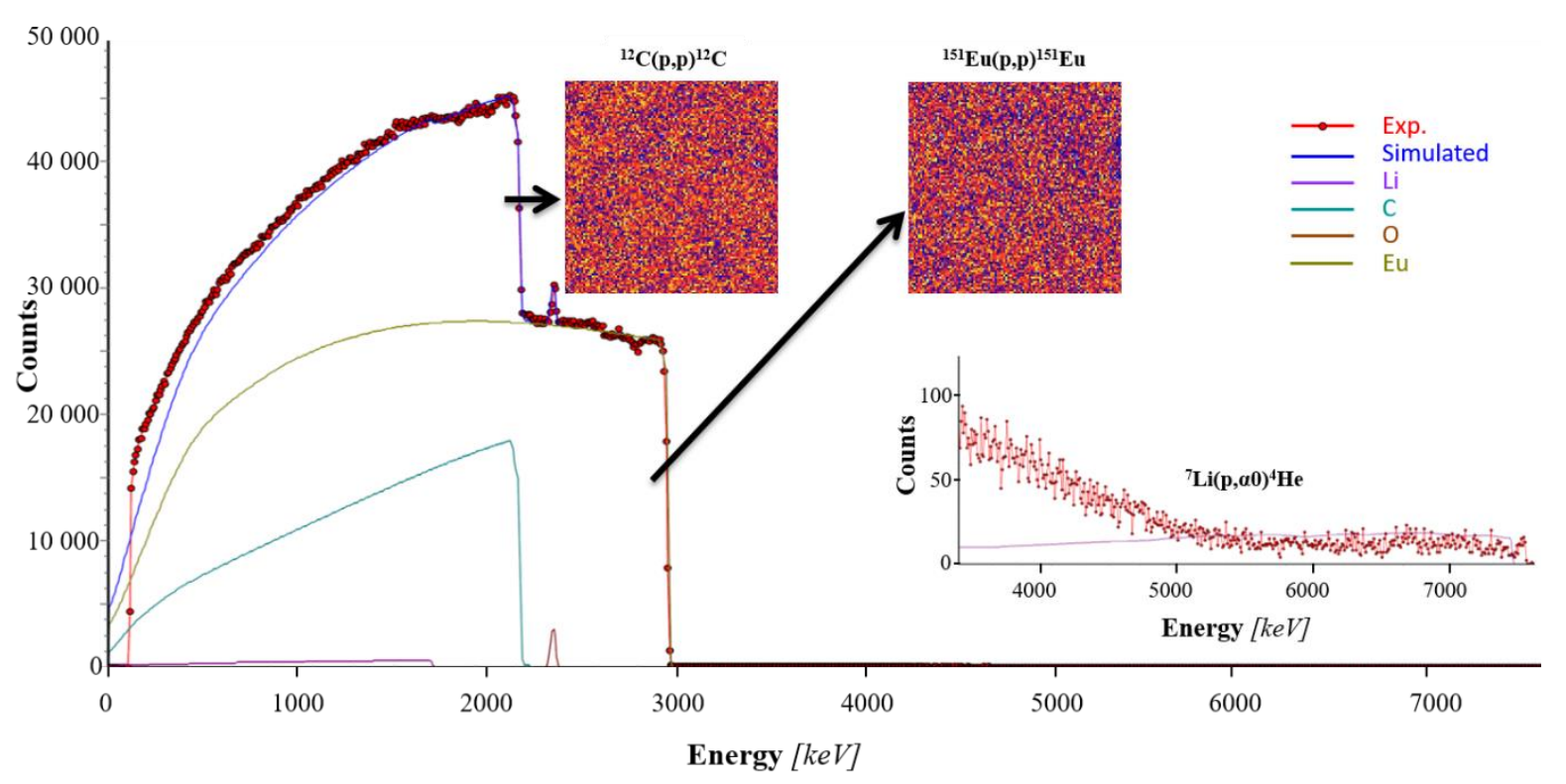

Figure 3 - Recorded RBS/NRA spectrum of the $\mathrm{EuC}_{6}$ GIC synthesized by the molten salts method and its simulation. Each individual elementary contribution to the simulation is drawn. Square maps represent intensity contrasts within narrow region of interest in order to enhance Eu and $\mathrm{C}$ contributions. Red spots: experiment; blue solid line: simulation. The inset is focused on the ${ }^{7} \mathrm{Li}$ nuclear reaction energy range.

First of all, several spectra have been recorded and Fig.3 corresponds to a spectrum representative of the sample. Regarding the maps recorded at the region of interest of europium and carbon, it clearly appears that the sample is homogeneous in depth and laterally for these both elements. The signal measured for the highest energy range of the spectrum due to the ${ }^{7} \mathrm{Li}\left(\mathrm{p},{ }^{4} \mathrm{He}\right){ }^{4} \mathrm{He}$ nuclear reaction highlights a very weak contribution of lithium. In the lower energy range, a small peak due to the ${ }^{16} \mathrm{O}(\mathrm{p}, \mathrm{p}){ }^{16} \mathrm{O}$ is observed at $2340 \mathrm{keV}$ whereas no significant contribution due to potassium or chlorine has been observed on spectra, that it to say that we prepared a GIC intercalated into the bulk, without any metal chloride inclusion.

As the ion beam analysis allows probing the sample laterally and in depth, even if some surface degredation occurs during the transfer to the analytic device, nuclear microprobe instrument constitutes a nice tool for understanding the homogenity of the GIC. Moreover the analysis areas are sufficiently large $\left(250 \times 250 \mu \mathrm{m}^{2}\right)$ to be representative of the sample. So, the maps corresponding to the elementary repartition of carbon and europium are presented on Fig.3, and are clearly uniform, confirming the successful intercalation of europium into the bulk, without any elementary over-concentration, gradient or depletion. This good homogeneity was not foreseen and constitutes a significant advantage in respect with the lithium-based molten alloy 
technique previously employed to synthesize $\mathrm{EuC}_{6}$ [29]. Indeed, in the latter case, ion beam analyses revealed that the sample was not totally homogeneous [30]. Regarding the homogeneity of the sample deduced from the elementary maps, it has been possible to simulate its composition on the whole analysis window. The general chemical composition is in agreement with the expected $\mathrm{EuC}_{6}$ composition deduced from the $\mathrm{XRD}$ pattern or the electronic density profile.

The sample has been simulated considering the superimposition of three slices with different thickness and composition. A first slice at the surface of the sample includes carbon, oxygen and lithium in its composition, due to an oxidizing phenomenon occuring during air exposure when the sample is transfered to the microprobe analysis device; a second one is only composed of carbon and oxygen. These slices are of course not taken in consideration to determine the elementary composition of the GIC as they are very thin (less than $1 \%_{\text {vol. }}$ ) and due to the migration of lithium (alkali metal displays an important affinity towards oxygen) toward surface during the transfer under air. Under this very thin oxidized film, a bulk sample without heterogenity has been modeled with the $\mathrm{EuC}_{6}$ composition. The very weak nuclear reaction between proton beam and lithium is interpreted as the presence of less than $2 \%$ at. in the sample, of course not detected by XRD. The presence of this element is clearly in relation with the presence of $\mathrm{LiCl}$ in the reactive mixture. In order to minimize this amount of lithium, precautions must be taken such as increasing the reaction time. Anyway, this amount is extremely low and is comparable to the one already pointed out for $\mathrm{CaC}_{6}$ prepared using the lithium-based molten alloy method $[39,41]$. Indeed, superconducting $\mathrm{CaC}_{6}$ samples include about $1 \%$ at. of lithium, but this is clearly negligeable regarding the subsequent analyses realized on these samples where lithium, present in a so weak amount, clearly does not perturbate the physical properties of $\mathrm{CaC}_{6}$.

The high level of purity of the synthesized samples was further confirmed by dc magnetometry. We performed measurements of temperature dependent magnetic susceptibility and isothermal magnetization by applying an external magnetic field parallel to the sample surface $(\vec{H} \perp \hat{c})$. The temperature dependent magnetic susceptibility is fully detailed in the SM. Very briefly, it presents three main features: first, our data confirmed the antiferromagnetic Néel ordering temperature $\left(\mathrm{T}_{\mathrm{N}}\right)$ at about $40 \mathrm{~K}$, field independent. Secondly, the high temperature susceptibility $\left(\mathrm{T}>\mathrm{T}_{\mathrm{N}}\right)$ is dominated by a paramagnetic-like behavior due to an effective magnetic moment of 7.2 $\mu \mathrm{B}$, which is quite close to $7.94 \mu_{\mathrm{B}}$, the value expected for this compound where the intercalated $\mathrm{Eu}$ is supposed to be divalent. Third, a small thermal hysteresis is present below 
about $25 \mathrm{~K}$ that is fully removed at high field and whose origin will be justified in the next paragraph.

More intriguing is the low-T field behavior of the isothermal magnetization. In Fig.4 we report the field dependent magnetization measured at several temperatures, spanning from 5 up to $50 \mathrm{~K}$ is presented. We can notice some important features:

i) the $\mathrm{EuC}_{6}$ phase diagram can be divided into four regions: (1) a spin-triangular antiferromagnetic ground state $\left(\mathrm{H}_{\mathrm{C} 0}<22 \mathrm{kOe}\right)$. (2) The ferrimagnetic plateau $\left(\mathrm{H}_{\mathrm{C} 0}=22 \mathrm{kOe}<\right.$ $\mathrm{H}<82 \mathrm{kOe}=\mathrm{H}_{\mathrm{C} 1}$ ) where the magnetization is roughly constant at about $1 / 3$ of the $\mathrm{Eu}^{2+}$ saturated magnetic moment $\left(\mathrm{S}=7 / 2 ; \mathrm{M}_{\mathrm{S}}=7 \mu_{\mathrm{B}}\right)$. (3) The canted state, where the magnetization increases linearly with the applied field up to $\mathrm{H}_{\mathrm{C} 2} \approx 205 \mathrm{kOe}$ and (4) the ferromagnetic region above $\mathrm{H}_{\mathrm{C} 2}$ where the magnetization is saturated to the full moment of the divalent europium. Since $55 \mathrm{kOe}$ is the maximum field available for our magnetometer, we can span just above $\mathrm{H}_{\mathrm{C} 0}$. In the 0-55 kOe field region the measured magnetization shows the expected first metamagnetic transition between 10 and $30 \mathrm{kOe}$. Both the amplitude of the hysteresis loop and the magnetization intensity in the ferrimagnetic plateau decrease with increasing temperature leaving place to a pure paramagnetic behavior for $\mathrm{T}>\mathrm{T}_{\mathrm{N}}$. This general behavior is fully in agreement with what was found in previous studies [42].

ii) The ferrimagnetic plateau measured at $5 \mathrm{~K}$ shows a saturation value of about $1.9 \mu_{\mathrm{B}}$ that is somehow lower than the expected value of $1 / 3$ of $\mathrm{M}_{\mathrm{S}}\left(\mathrm{Eu}^{2+}\right)$. This effect could be justified by the presence of a small percentage of trivalent europium diluted in the pure phase.

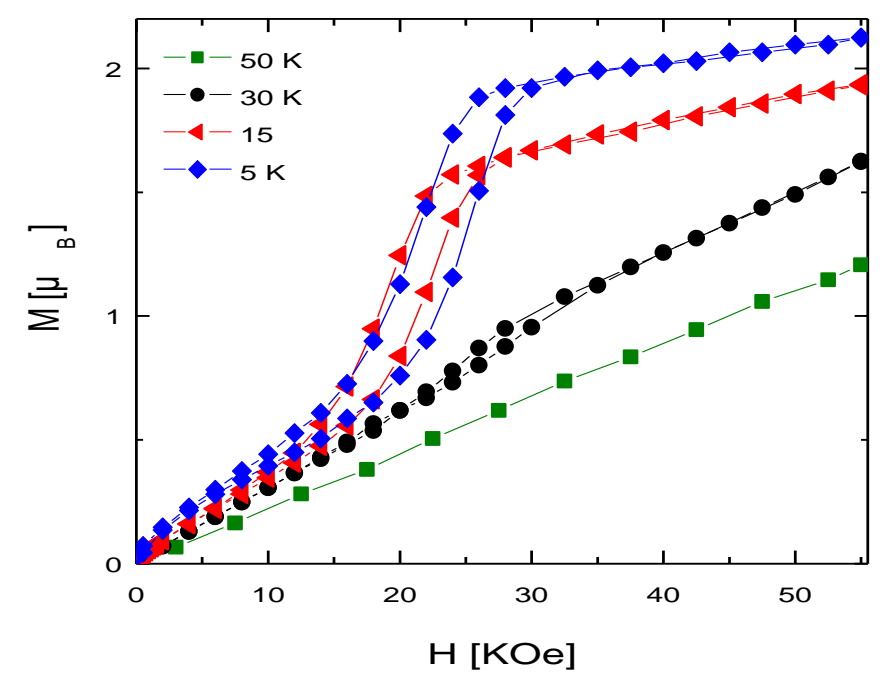

Figure 4 - Field dependence of the magnetic moment of the $\mathrm{EuC}_{6}$ sample at $\mathrm{T}=5,15,30$ and $50 \mathrm{~K}$. 


\section{Conclusion}

The $\mathrm{LiCl}-\mathrm{KCl}$ eutectic molten salt in which metallic europium was dissolved at $450{ }^{\circ} \mathrm{C}$ was used in order to perform intercalation reactions. Experimental parameters have been empirically investigated and favorable conditions to obtain the $\mathrm{EuC}_{6}$ binary GIC intercalated into the bulk have been pointed out. Using this method, the obtained GIC is much more homogeneous than $\mathrm{EuC}_{6}$ samples prepared by other techniques. We deduced from this first study that this original way of synthesis is of great interest in order to prepare homogenous bulk GIC samples, especially for metals whose affinity for graphite is not significant. This result opens new and very large perspectives for the intercalation of electron donors into graphite.

\section{Acknowledgments}

The authors want to thank Camille Thevenot and Pierre Venturini for preliminary work in this study. Jean-François Marêché is also greatly thanked for its technical support for initiating this project. The technical team of the nuclear microprobe instrument of the NIMBE laboratory (CEA) has been precious in order to realize ion beam measurements and we thank them by these few words. G.L. thanks Prof. F. Canepa for fruitful discussions and suggestions.

\section{References}

[1] Dresselhaus MS, Dresselhaus G. Intercalation compounds of graphite, Adv. Phys. 51(1) (2002) 1-186.

[2] Enoki T, Suzuki M, Endo M. Graphite intercalation compounds and applications, Oxford University Press: New York, (2003) 1-456.

[3] Matsumoto R, Okabe Y. Highly electrically conductive and air-stable metal chloride ternary graphite intercalation compounds with $\mathrm{AlCl}_{3}-\mathrm{FeCl}_{3}$ and $\mathrm{AlCl}_{3}-\mathrm{CuCl}_{2}$ prepared from flexible graphite sheets, Synth. Met. 222(B) (2016) 351-5.

[4] Matsumoto R, Okabe Y. Electrical conductivity and air stability of $\mathrm{FeCl}_{3}, \mathrm{CuCl}_{2}, \mathrm{MoCl}_{5}$, and $\mathrm{SbCl}_{5}$ graphite intercalation compounds prepared from flexible graphite sheets, Synth. Met. 212 (2016) 62-8. 
[5] Kazuhiko M, Takao I. Magnetic susceptibility measurements on Li-intercalateed graphite: Paramagnetic to diamagnetic transitions in $\mathrm{C}_{12} \mathrm{Li}$ induced by magnetic field, Carbon 123 (2017) 645-650.

[6] Lamura G, Shiroka T, Cahen S, Rida H, Emery N, Marêché J-F et al. The zero-field magnetic ground state of $\mathrm{EuC}_{6}$ investigated by muon spectroscopy. Carbon 50 (2012) 39954001.

[7] Shiroka T, Thede M, Wittenfeld L, Litterst FJ, Cahen S, Rida H et al. Competing magnetic interactions in the graphite-intercalation compound $\mathrm{Li}_{0.25} \mathrm{Eu}_{1.95} \mathrm{C}_{6}$, Carbon 63 (2013) 294-302. [8] Tkachuk VY, Ovsiyenko IV, Matzui LY, Len TA, Prylutskyy YI, Brusylovets OA, et al. Asymmetric magnetoresistance in the graphite intercalation compounds with cobalt, Mol. Cryst. Liq. Cryst. 639(1) (2016) 137-50.

[9] Smith RP, Weller TE, Howard CA, Dean MPM, Rahnejat KC, Saxena SS et al. Superconductivity in graphite intercalation compounds, Physica C: Superconductivity and Its Applications 514 (2015) 50-8.

[10] Weller TE, Ellerby M, Saxena SS, Smith RP, Skipper NT. Superconductivity in the intercalated graphite compounds $\mathrm{C}_{6} \mathrm{Yb}$ and $\mathrm{C}_{6} \mathrm{Ca}$, Nat. Phys. 1 (2005) 39-41.

[11] Ellerby M, Weller TE, Saxena SS, Smith RP, Skipper NT. Superconductivity at elevated temperatures in $\mathrm{C}_{6} \mathrm{Yb}$ and $\mathrm{C}_{6} \mathrm{Ca}$, Physica B 378-380 (2006) 636-639.

[12] Castro DD, Kanigel A, Maisuradze A, Keren A, Postorino P, Rosenmann D et al. Muon spin rotation study of the magnetic penetration depth in the intercalated graphite superconductor CaC6, Phys. Rev. B 82 (2010) 0145301-6.

[13] Abdelkader AM, Cooper AJ, Dryfe RAW, Kinloch IA. How to get between the sheets: a review of recent works on the electrochemical exfoliation of graphene materials from bulk graphite, Nanoscale 7(16) (2015) 6944-56.

[14] Noel M, Santhanam R. Electrochemistry of graphite intercalation compounds, J. Power Sources 72(1) (1998) 53-65.

[15] Bottomley MJ, Parry GS, Ubbelohde AR, Young DA. Electrochemical preparation of salts from well-oriented graphite, J. Chem. Soc. (Dec.) (1963) 5674-80.

[16] Inagaki M. New development in alkali metal-graphite intercalation compounds, Tanso 170 (1995) 298-306.

[17] Guérard D, Nalimova VA. Synthesis of graphite intercalation compounds with donor species, MRS Symposium Proceedings 369(Solid State Ionics IV) (1995) 155-63.

[18] Hick SM, Griebel C, Blair RG. Mechanochemical synthesis of alkaline earth carbides and intercalation compounds, Inorg. Chem. 48 (2009) 2333-8. 
[19] Hwang DM, Solin SA, Guérard D. The Raman spectrum of EuC 6 : a clue to understanding light scattering from stage 1 alkali GICs, Physics of Intercalation Compounds (1981) 187-92. [20] El Makrini M, Guérard D, Lagrange P, Hérold A. Intercalation of rare earth metals in graphite, Physica 99B (1980) 481-5

[21] Hwang DM, Guérard D, Raman scattering in stage one europium intercalated graphite, Solid State Comm. 40(7) (1981) 759-64.

[22] El Makrini M, Guérard D, Lagrange P, Hérold A. Insertion de lanthanoïdes dans le graphite, Carbon 18 (1980) 203-9.

[23] Emery N, Hérold C, Lagrange P. The synthesis of binary graphite-metal intercalation compounds using molten lithium alloys, Carbon 46(1) (2008) 72-5.

[24] Emery N, Hérold C, Lagrange P. Overview on the intercalation reactions of lithium alloys into graphite, Prog. Solid State Chem. 36(3) (2008) 213-22.

[25] Pruvost S, Hérold C, Hérold A, Lagrange P. Structural study of novel graphite-lithiumcalcium intercalation compounds, Eur. J. Inorg. Chem. 8 (2004) 1661-7.

[26] Emery N, Hérold C, d'Astuto M, Garcia V, Bellin Ch, Marêché J-F, et al. Superconductivity of bulk $\mathrm{CaC}_{6}$, Phys. Rev. Lett. 95 (2005) 87003.

[27] Emery N, Hérold C, Marêché J-F, Bellouard C, Loupias G, Lagrange P. Superconductivity in $\mathrm{Li}_{3} \mathrm{Ca}_{2} \mathrm{C}_{6}$ intercalated graphite, J. Solid State Chem. 179 (2006) 1289-92.

[28] Emery N, Hérold C, Bellouard C, Delcroix P, Marêché J-F, Lagrange P. Synthesis and characterisation of a novel europium-based graphite intercalation compound, J. Solid State Chem. 181 (2008) 2924-9.

[29] Rida H, Cahen S, Hérold C, Lagrange P. Bulk synthesis and crystal structure of the first stage europium-graphite intercalation compound, Carbon, 48 (2010) 3190-5.

[30] Cahen S, Rida H, Berger P, Fauchard M, Lagrange P, Marêché J-F, et al. Graphite-lithiumeuropium system: modulation of the structural and physical properties of the lamellar phases as a consequence of their chemical composition, Carbon 77 (2014) 803-13.

[31] Hagiwara R, Ito M, Ito Y. Graphite intercalation compounds of lanthanides metals prepared in molten chlorides, Carbon 34 (1996) 1591-3.

[32] Basin AS, Kaplun AB, Meshalkin AB, Uvarov NF. The LiCl-KCl binary system, Russ. J. of Inorg. Chem. 53(9) (2008) 1509-11.

[33] Leung SY, Dresselhaus MS, Underhill C, Krapchev T, Dresselhaus G, Wuensch BJ. Structural studies of graphite intercalation compounds using $00 l$ x-ray diffraction, Phys. Rev. B 24 (1981) 3505-18. 
[34] Mayer M. SIMNRA, a simulation program for the analysis of NRA, RBS and ERDA. In: Duggan JL and Morgan IL, editor, Proceedings, 15th international conference on the application of accelerators in research and industry. AIP conference proceedings 475 (1999) 541.

[35] Meyer, G. ; Institut fur Anorganische Chemie, Universitat Hannover, Hannover, Germany. ICDD Grant-in-Aid, 1990.

[36] Kaindl G, Feldhaus J, Ladewig U, Frank KH. Electronic and magnetic properties of europium-intercalated graphite, Phys. Rev. Lett 50 (1983) 123-6.

[37] Wortmann G. Synthetic metals: applications of the Mössbauer effect and other methods, Hyperfine Interact. 27(1-4) (1986) 263-77.

[38] Cookson JA, The nuclear microprobe and competing techniques, Nucl. Instr. Meth. Phys. Res. B 54 (1991) 433-440.

[39] Pruvost S, Berger P, Hérold C, Lagrange P. Nuclear microanalysis: an efficient tool to study intercalation compounds containing lithium, Carbon 42 (2004) 2049-56.

[40] Wang Y and Nastasi M. Handbook of Modern Ion Beam Materials Analysis, Cambridge University Press (2010)

[41] Berger P, Pruvost S, Hérold C, Lagrange P. Proton enhanced scattering and nuclear reaction analysis microcharacterization of ternary graphite-lithium-calcium intercalation compounds, Nucl. Instr. Meth. Phys. Res. B 219-220 (2004) 1005-9.

[42] Suematsu H, Ohmatsu K, Yoshizaki R. Magnetic properties of Europium-graphite intercalation compound $\mathrm{C}_{6} \mathrm{Eu}$, Sol. State Com. 38 (1981) 1103-7. 